\title{
Hybrid FDTD and Single-Scattering Theory for Simulation of Scattering From Hard Targets Camouflaged Under Forest Canopy
}

\author{
Kamal Sarabandi, Fellow, IEEE, Mojtaba Dehmollaian, Student Member, IEEE, and \\ Hossein Mosallaei, Senior Member, IEEE
}

\begin{abstract}
A hybrid target-foliage model is developed to investigate the scattering behavior of hard targets embedded inside a forest canopy. The proposed model is composed of two existing electromagnetic-scattering models, one for the foliage and the other for the hard targets that are coupled in a computationally efficient manner. The connection between these two models, which accounts for the interaction between the foliage scatterers and the target, is accomplished through the application of Huygens' principle. Wave penetration through the forest canopy and near-field and far-field scattering from its constituents is calculated using a coherent single-scattering theory, which makes use of realistic tree structures. Defining a Huygens' surface enclosing the hard target and calculating the illuminating field (the scattered fields from the nearby vegetation scatterers and reduced incident field), the interaction between the foliage and the hard target is accounted for. Computing the scattered field from target on the Huygens' surface and using a reciprocity theorem target-foliage interaction is captured very efficiently. Calculation of scattering from a hard target is carried out using a finite-difference time-domain (FDTD) technique. For a typical vehicle dimensions, the required time and memory for the FDTD computation and exact field calculation inside the foliage limits the simulation frequency to upper very high frequency (VHF) band.
\end{abstract}

Index Terms-Electromagnetic (EM) scattering, finitedifference time-domain (FDTD) method, hybrid solution methods.

\section{INTRODUCTION}

D ETECTION and identification of hard targets inside vegetation canopies have long been a challenging problem in the area of remote sensing. Development of theoretical and numerical models for this problem has a wide range of both civilian and military applications. The ability of electromagnetic (EM) waves to penetrate through the foliage at low frequencies has made synthetic aperture radars (SARs) operating at the very high frequency (VHF) band ideal tools for detection

Manuscript received May 26, 2005; revised November 3, 2005. This work was supported by the Army Research Office under Contract DAAD19-02$1-0262$.

K. Sarabandi and M. Dehmollaian are with the Radiation Laboratory, Departmet of Electrical Engineering and Computer Science, University of Michigan, Ann Arbor, MI 48109 USA (e-mail: saraband@umich.edu).

H. Mosallaei was with the Radiation Laboratory, Department of Electrical Engineering and Computer Science, University of Michigan, Ann Arbor, MI 48109 USA. He is now with the Department of Electrical and Computer Engineering, College of Engineering, Northeastern University, Boston, MA 02115 USA.

Digital Object Identifier 10.1109/TGRS.2006.872091 of targets in the foliage [1], [2]. However, the scattering effect of the tree constituents can distort the signal phase front, reducing the SAR resolution and changing the target signature. In this regard, a comprehensive phenomenological study is needed to accurately investigate the EM interaction of the foliage and an arbitrary hard target and vice versa. The literature concerning modeling the propagation through and scattering from forest canopies are many [3]-[7]. However, the problem of accurate modeling of scattering from hard targets embedded inside tree canopies remains an unsolved problem [8], [9]. The difficulties associated with developing such model are threefold: one is the accurate computation of the field inside a forest; the second is characterization of the scattered field from the target illuminated by the field inside the forest; and the third is the computation of the scattered-field interaction with the foliage, and the total scattered field outside the forest at the observation point.

Considering the size of tree structures, the aspect ratio of the dimension of its constituent particles, the variability in dielectric constant of scatterers, and the relatively large number of trees around a target, brute force computational methods are not suitable for this problem. In addition, applicability of exact numerical methods is limited to very low frequencies where scattering from leaves and branches can be totally ignored. However, vegetation canopies can be viewed as a sparse random medium, having a volume fraction typically less than $1 \%$. For such media, multiple scattering among constituent scatterers is of secondary importance and can be ignored. In this case, EM scattering from vegetation canopies can be simplified in terms of problems of scattering from individual dielectric cylinders and thin dielectric disks, modeling branches and leaves, arranged in a semideterministic fashion. The formulations for calculating the scattering and attenuation caused by vegetation particles have been derived analytically, using high- and lowfrequency techniques [3], [4]. The effect of the ground plane is also accounted for, through the application of image theory maintaining only the saddle point contribution. For precise prediction of the field behavior inside the canopy, the structure of the trees is preserved in the forest model. This is done using a fractal-based model [known as Lindenmayer systems (Lsystems)], which can generate very complex tree structures with a finite number of structural and botanical parameters. Monte Carlo coherent single-scattering model, which makes use of fractal-generated trees, has been proposed in [10] and [11] 
to estimate the scattering from and propagation through the forest canopy. In this model, the coherent summation of all scattering contributions, including those from the ground plane, is computed to find the total field at a given point within the forest canopy. This total field is used as the excitation source for the hard target.

For the problem of EM scattering from an isolated target of arbitrary shape, a number of approximation and numerical approaches may be used. In VHF band (20-200 MHz), highfrequency approximation techniques are not usually applicable for typical ground vehicles, where the wavelength is comparable to the vehicle dimensions. Therefore, exact numerical approaches such as method of moments (MoM) or finitedifference time-domain (FDTD) are appropriate to treat the scattering problem. In past, FDTD technique has been widely used for simulation of scattering for a wide range of scattering problems [12]-[15]. To get the benefit of FDTD, accurate analysis for complex targets, and yet to account for scattering interaction with a nearby vegetation in a computationally tractable manner, we use a hybridization of FDTD with the coherent single-scattering model for the forest.

The main contribution of this paper is in the construction of a foliage-target model that is computationally tractable and can provide accurate results. This model accounts for scattering from the foliage, hard target, and near-field interaction of the foliage and the hard target. In what follows, first, we derive a general formulation for backscattering from a target above the ground plane and embedded in the foliage, illuminated by a plane wave of arbitrary polarization. This formulation makes use of the forest model for computation of the near-field scattering from the trees over a Huygens' surface enclosing the target and a full-wave FDTD formulation for a computation of the scattered field from the target on the same Huygens' surface. The reciprocity is used to compute the radiated field from the Huygens' surface at the observation point [12], [14]. The model is validated using an iterative physical optics (IPOs) [16]-[18] in conjunction with physical theory of diffraction (PTD) solution applied to a dihedral corner reflector in free space and above the dielectric half-space. Having the hybrid model (FDTD + Huygens' surface approach + reciprocity) validated, the model is then used to simulate the polarimetric radar response of a metallic vehicle under the foliage.

\section{THEORY}

In this section, the basic formulation of the proposed hybrid time- and frequency-domain method for studying an accurate radar backscatter response of a foliage-camouflaged targets is presented. The overall model is composed of three basic components: 1) a frequency-domain forest model; 2) FDTD model for the hard target; and 3) an algorithm that interfaces the forest and target models and allows for computation of targetfoliage-scattering interaction in an efficient manner.

\section{A. Coherent Forest Model}

For developing an EM-scattering method for detection and identification of hard targets embedded inside vegetation canopies, a comprehensive physics-based model is needed.
Such a model must be able to preserve the phase of the backscatter so that SAR, interferometric SAR, and polarimetric SAR simulations are possible. In the single-scattering theory, field computation is carried out coherently, to preserve the phase of the scattered fields. Such a model is implemented in [10] where the scattering from each tree component, when illuminated by the mean field, is calculated and the total scattering is expressed as the coherent summation of all the scattering contributions. This model is capable of an accurate estimation of the polarimetric radar response from forested environments. In the following, we review the basic concept and mathematical formulations used in this model [7], [19].

It has been demonstrated that tree structures have significant effects on the radar backscatter response of the tree canopy as well as propagation behavior of the signal going through the forest [20]. Implementation of the L-system, using fractal theory, has shown to be an effective approach for including the tree structures in the EM-scattering model [21]-[23]. Basically, in the L-system, the structures of the trees are generated through a rewriting process [24], using a finite number of botanical and geometrical parameters. In this model, tree constituent components such as branches and leaves are represented by dielectric cylinders and thin dielectric disks, respectively, and arranged in a semideterministic fashion. Then, the single-scattering theory based on distorted Born approximation is used, where for every scatterer inside the forest illuminated by the mean-field, the scattered field is computed at the observation point and the total scattered field is expressed as a coherent sum of all scattering contributions. Therefore, the total scattered field $\bar{E}^{s}$ can be mathematically expressed as

$$
\bar{E}^{s}=\frac{e^{i k r}}{r} \sum_{n=1}^{N} e^{i \phi_{n}} S_{n} \cdot \bar{E}_{n}^{i}
$$

where $N$ is the total number of scatterers, $S_{n}$ is the scattering matrix of the $n$th scatterer, and $\phi_{n}$ is a path delay phase given by $\phi_{n}=\left(\hat{k}_{i}-\hat{k}_{s}\right) \cdot \bar{r}_{n}$. Here, $\bar{r}_{n}$ is a vector denoting the location of the $n$th scatterer, and $\hat{k}_{i}$ and $\hat{k}_{s}$ are the propagation unit vectors along the direction of incident and scattered fields, respectively. The scattering from tree constituent components $S_{n}$ is calculated in the presence of a dielectric half-space representing the ground plane. Using a geometric optics approximation, scattering from each scatterer can be decomposed into four components: 1) direct $S_{n}^{\mathrm{t}}$;2) ground-scatterer $S_{n}^{\mathrm{tg}}$; $3)$ scatterer-ground $S_{n}^{\text {gt }}$; and 4) ground-scatterer-ground $S_{n}^{\text {gtg }}$. Therefore, the scattering matrix $S_{n}$ can be written as

$$
S_{n}=S_{n}^{\mathrm{t}}+S_{n}^{\mathrm{gt}}+S_{n}^{\mathrm{tg}}+S_{n}^{\mathrm{gtg}} .
$$

The model presented in [10] makes use of far-field expressions for scattering matrices used in (2). This model is used to compute the backscattered field from the foliage alone in the far-field region. A more advanced model was developed in [11], where the observation point can be placed inside the forest, possibly in the near field of some scatterers. Scattering formulations for individual scatterers were modified to achieve a uniform scattering solution for scatterers valid from nearfield to far-field regions. For the computation of the scattered 
field at a point inside the forest, distorted Born approximation is invoked [7]. That is, each scatterer is assumed to be inside an effective homogeneous medium and illuminated by a mean field, composed of two components: 1) direct and 2) reflected from the ground. Therefore, in (1), $\bar{E}_{n}^{i}$ is the mean field calculated at each scatterer location and similarly, in the calculation of the scattering matrix in (2), the presence of such equivalent medium is considered.

Calculation of the mean field is carried out using Foldy's approximation [7]. According to Foldy's approximation, inside a uniform random medium the coherent part of the field (mean field) $\bar{E}$, has the following variation with respect to the distance $s$ along the propagation direction $\hat{k}$ :

$$
\frac{d \bar{E}}{d s}=i K \cdot \bar{E}
$$

where

$$
K=\left[\begin{array}{cc}
k_{0}+M_{v v} & M_{v h} \\
M_{h v} & k_{0}+M_{h h}
\end{array}\right]
$$

and

$$
M_{p q}=\frac{2 \pi n_{0}}{k_{0}}\left\langle S_{p q}^{0}(\hat{k}, \hat{k})\right\rangle .
$$

In (5), $n_{0}$ is the volume density of the scatterers, $k_{0}$ is the propagation constant in free space, and $\left\langle S_{p q}^{0}(\hat{k}, \hat{k})\right\rangle$ is the ensemble average of the forward-scattering matrix, where $p$ and $q$ can be $v$ or $h$ for vertical or horizontal polarizations, respectively. Solution for (3), is given by $\bar{E}=e^{i k_{0} s} T(s, \hat{k}) \cdot \bar{E}^{0}$, where $\bar{E}^{0}$ is the field at $s=0$. Matrix $T$ is called the transmissivity matrix, and is given by

$$
T=\left[\begin{array}{ll}
e^{i M_{v v} s} & e^{i M_{v h} s} \\
e^{i M_{h v} s} & e^{i M_{h h} s}
\end{array}\right] .
$$

It is noted that the imaginary part of $M_{p q}$, called extinction coefficient, accounts for the extinction of the mean field propagating inside the random medium. This is due to the scattering and absorption caused by random particles. To account for variability in particle shape and distribution along the vertical extent of the forest stand, the random medium (forest stand) is divided into $M$ homogeneous strata with different effective dielectric constants. Based on Foldy's approximation (5), the propagation constant of each layer is proportional to the ensemble average of the forward-scattering-matrix elements of scatterers inside that layer [7]. It is noted that the boundaries between the layers are diffuse where no reflection or refraction take place. For determining the number of layers $M$, the code starts with a moderate number of layers. Then, the extinction of each layer is calculated. If the discontinuity of extinction between any two layers is bigger than a predefined threshold, then the forest stand is divided in to finer layers.

Fig. 1 shows the simulate extinction profile of a pine stand with a tree number density of 0.05 trees $/ \mathrm{m}^{2}$ versus height at two different frequencies and polarizations. As shown, we observe higher attenuation coefficient for higher frequency than that of lower frequency due to the scattering and absorption losses of

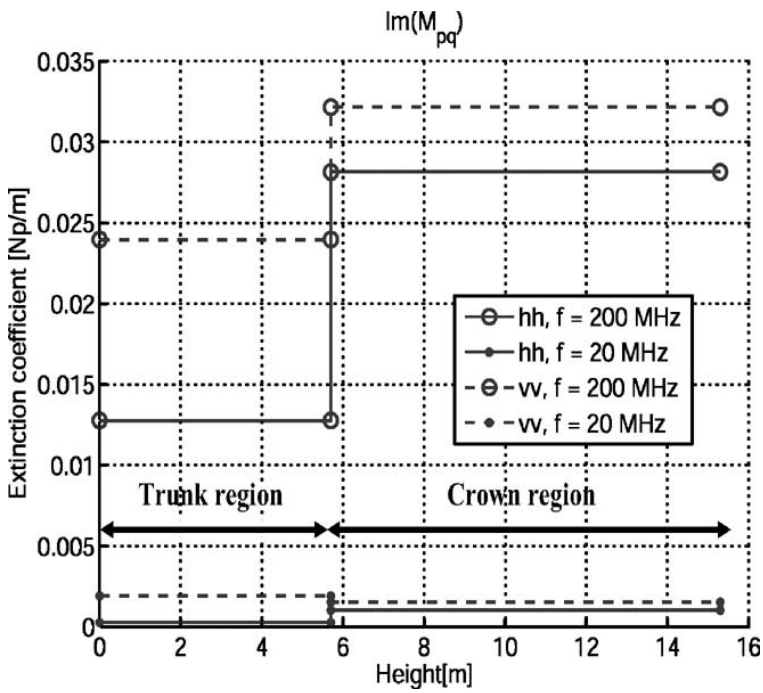

Fig. 1. Extinction profile of pine tree stand with density of 0.05 trees $/ \mathrm{m}^{2}$ for incidence elevation angle of $30^{\circ}$.

vegetation particles. According to Rayleigh scattering, at low frequencies the scattering matrix $S_{p q}^{0}(\hat{k}, \hat{k})$ is proportional to $k_{0}^{2}$ [25]. Therefore, according to (5), the extinction coefficient is proportional to $k_{0}$. As shown in Fig. 1, it is expected that extinction at $200 \mathrm{MHz}$ be about ten times higher than that at $20 \mathrm{MHz}$. In addition, vertical polarization of incident field has a higher extinction coefficient than horizontal polarization because of the specific arrangement of scatterers in the tree structure. Due to higher volume density of scatterers, the crown region shows the higher extinction coefficient than trunk region except for very low frequencies where vertical polarization experiences slightly more attenuation in trunk region compared to the crown region.

\section{B. Computation of Local Scattered Field Using FDTD}

The FDTD technique is a very versatile full-wave numerical approach appropriate for targets of arbitrary geometry and material properties and for characterization of the scattered field over a wide range of frequencies [26], [27]. Although FDTD formulation is very general, for a very large computation domain such as the foliage-camouflaged target, the computation time and the required memory become prohibitively large [14]. Another level of difficulty in the brute force application of FDTD is the aspect ratio of the dimensions of scatterers such as broad leaves and needles. Because of these difficulties the computational domain is limited to the target itself excluding the surrounding foliage. In order to calculate the scattered field from the target using FDTD, the computational domain is divided into the total-field and scattered-field regions separated by a virtual interface enclosing the target. This interface serves as a connecting boundary over which the difference between the total field and the scattered field (incident field) is imposed [26]-[28]. The target structure is located inside the total field region surrounded by the virtual rectangular box. Using the forest model, the field on the FDTD box is computed and considered as the incident field for the target. Using the FDTD scheme, the scattered field by the target itself is calculated at 
the same grid points on the box, and then using a scheme based on the reciprocity theorem, the backscattered field at the radar position is computed.

\section{Computation of Backscattered Field}

The backscatter field from a target inside the forest canopy is composed of two main components. The first component is the direct scattering from the target simply attenuated by the foliage above, and second component contains the scatteredfield interaction between the foliage and the target as well as the target and the foliage. In order to calculate the backscattered field from a target inside the forest canopy without the computation of scattering interaction of target radiated field and the surrounding foliage, the reciprocity theorem can be used. The application of the reciprocity for a computation of first-order-scattering interaction between two objects has been demonstrated in past [29]. This method in conjunction with a Huygens' surface is used here to account for an interaction of a random media with a complex hard target. According to the reaction theorem, for two sets of sources and fields, denoted by 1 and 2, we have [30]

$$
\int_{V_{2}}\left(\bar{E}_{1} \cdot \bar{J}_{2}-\bar{H}_{1} \cdot \bar{M}_{2}\right) d \mathbf{r}=\int_{V_{1}}\left(\bar{E}_{2} \cdot \bar{J}_{1}-\bar{H}_{2} \cdot \bar{M}_{1}\right) d \mathbf{r}
$$

where $\bar{E}$ and $\bar{H}$ are electric and magnetic fields, and $\bar{J}$ and $\bar{M}$ are the electric and magnetic current sources, respectively. $\bar{E}_{1}$ and $\bar{H}_{1}$ are fields generated by $\bar{J}_{1}$ and $\bar{M}_{1}$ at the location of the second sources $\bar{J}_{2}$ and $\bar{M}_{2}$ and similarly $\bar{E}_{2}$ and $\bar{H}_{2}$ are produced by $\bar{J}_{2}$ and $\bar{M}_{2}$ at $\bar{J}_{1}$ and $\bar{M}_{1}$ location. For problem at hand, we consider elementary current sources $\bar{J}_{1}$ and $\bar{M}_{1}$ at the radar location and induced currents on target as the secondary sources. Consider an elementary current source $\bar{J}_{1}$ at the radar position $\bar{r}_{\mathrm{R}}$, oriented along a polarization unit vector $-\hat{p}$ given by

$$
\bar{J}_{1}=\frac{4 \pi}{i k_{\mathrm{o}} Z_{\mathrm{o}}} \frac{r_{\mathrm{R}}}{e^{i k_{\mathrm{o}} r_{\mathrm{R}}}} \delta\left(\bar{r}-\bar{r}_{\mathrm{R}}\right)(-\hat{p}) .
$$

Here, $k_{\mathrm{o}}$ and $Z_{\mathrm{o}}$ are the propagation constant and characteristic impedance of the free space, respectively, and we assume $\bar{M}_{1}=0$. This source produces $\hat{p}$-polarized field at the far-field region. The unit vector $\hat{p}$ can be chosen $\hat{h}_{i}$ or $\hat{v}_{i}$ for horizontal or vertical polarization, respectively. These unit vectors in a global coordinate system are defined by

$$
\begin{gathered}
\hat{h}_{i}=\frac{\hat{k}_{i} \times \hat{z}}{\left|\hat{k}_{i} \times \hat{z}\right|} \\
\hat{v}_{i}=\hat{h}_{i} \times \hat{k}_{i}
\end{gathered}
$$

where $\hat{k}_{i}$ is the unit vector along the propagation direction of the incident field. Substituting (8) in (7), we have

$$
\bar{E}_{2} \cdot(-\hat{p})=\frac{i k_{\mathrm{o}} Z_{\mathrm{o}}}{4 \pi} \frac{e^{i k_{\mathrm{o}} r_{\mathrm{R}}}}{r_{\mathrm{R}}} \int_{V_{2}}\left(\bar{E}_{1} \cdot \bar{J}_{2}-\bar{H}_{1} \cdot \bar{M}_{2}\right) d \mathbf{r} .
$$

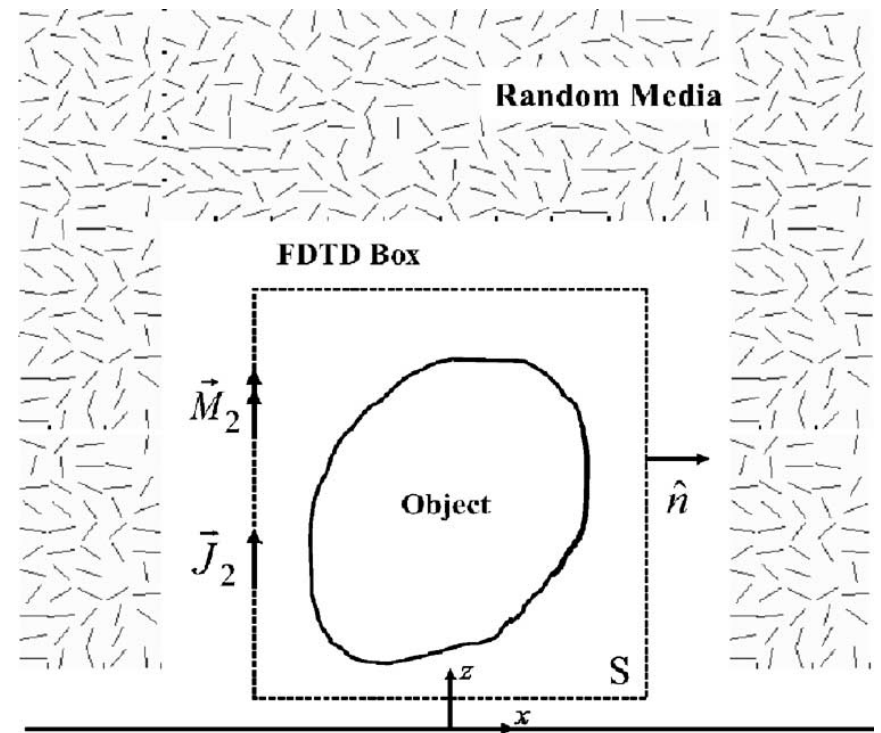

Ground Plane

Fig. 2. Geometry of the problem. Surface $\mathbf{S}$ is containing an object above the ground plane.

Hence, once the incident field and the induced currents on a target are known the backscattered field can be computed from (10). Using (10), each component of the scattering matrix can be computed from

$$
\mathbf{S}_{p q}= \pm \frac{i k_{\mathrm{o}} Z_{\mathrm{o}}}{4 \pi} \int_{V_{2}}\left(\bar{E}_{1 p} \cdot \bar{J}_{2 q}-\bar{H}_{1 p} \cdot \bar{M}_{2 q}\right) d \mathbf{r}
$$

where $p$ and $q$ represent the $h$ or $v$ polarizations. The first and second subscripts of scattering elements denote the receive and transmit polarizations, respectively. Since for backscatter direction $\hat{k}_{s}=-\hat{k}_{i}$, we have $\hat{h}_{s}=-\hat{h}_{i}$ and $\hat{v}_{s}=\hat{v}_{i}$, the minus sign in (11) is chosen when the receive polarization is vertical. Finally, the radar cross section (RCS) of the target is calculated from

$$
\sigma_{p q}^{s}=4 \pi\left|\mathbf{S}_{p q}\right|^{2}
$$

In (7), the electric and magnetic currents $\bar{J}_{2}$ and $\bar{M}_{2}$ are generally any equivalent electric and magnetic currents that can be directly computed on the target surface or on a hypothetical surface enclosing the target. These equivalent currents can produce the same scattered field as these produced by the physical induced currents. Using Huygens' principle, the tangential surface fields over a general closed surface that contains only the target (and not the foliage) are sufficient to generate the equivalent currents. Fig. 2 shows a fictitious rectangular box with surface $\mathbf{S}$ around the target. Denoting the scattered field outside $\mathbf{S}$ by $\bar{E}_{2}$ and $\bar{H}_{2}$, then according to Huygens' principle, we have

$$
\begin{aligned}
\bar{E}_{2}\left(\bar{r}^{\prime}\right)=\int_{\mathrm{S}}\left[i k_{\mathrm{o}} Z_{\mathrm{o}}(\hat{n}\right. & \left.\times \bar{H}_{2}(\bar{r})\right) \cdot \overline{\bar{G}}\left(\bar{r}, \bar{r}^{\prime}\right) \\
& \left.+\left(\hat{n} \times \bar{E}_{2}\right) \cdot \nabla \times \overline{\bar{G}}\left(\bar{r}, \bar{r}^{\prime}\right)\right] d s
\end{aligned}
$$


where $\overline{\bar{G}}\left(\bar{r}, \bar{r}^{\prime}\right)$, is the dyadic Green's function for the media, and $\hat{n}$ is the normal unit vector pointing outward. Note that for complex random media such as a forest canopy the exact computation of the Green's function is not possible and therefore the computation of the scattered field in the presence of the random media in a direct manner is not possible. The quantities $(\hat{n} \times$ $\left.\bar{H}_{2}\right)$ and $\left(-\hat{n} \times \bar{E}_{2}\right)$ can be interpreted as the equivalent electric and magnetic surface current densities, respectively, i,e.,

$$
\begin{aligned}
\bar{J}_{2}(\bar{r}) & =\hat{n} \times \bar{H}_{2}(\bar{r}) \\
\bar{M}_{2}(\bar{r}) & =-\hat{n} \times \bar{E}_{2}(\bar{r}) .
\end{aligned}
$$

Substituting (14) in (11), it can be shown that

$$
S_{p q}= \pm \frac{i k_{\mathrm{o}} Z_{\mathrm{o}}}{4 \pi} \int_{\mathbf{S}}\left[H_{2 q} \times \bar{E}_{1 p}+\bar{E}_{2 q} \times \bar{H}_{1 p}\right] \cdot \hat{n} d s .
$$

For our problem, the incident fields on the target $\bar{E}_{1}$ and $\bar{H}_{1}$ are composed of the superposition of the scattered fields from all tree constituents such as branches, trunks, and leaves, and the direct field attenuated by the foliage (mean field). Each of these field components can be decomposed into a direct and a reflected field from the ground plane. For a $\hat{p}$-polarized incident plane wave

$$
\begin{aligned}
& \bar{E}_{1 p}=\left(\bar{E}_{\mathrm{d}}+\bar{E}_{\mathrm{r}}\right)_{\text {mean }}+\sum_{\text {Scatterers }}\left(\bar{E}_{\mathrm{d}}+\bar{E}_{\mathrm{r}}\right) \\
& \bar{H}_{1 p}=\left(\bar{H}_{\mathrm{d}}+\bar{H}_{\mathrm{r}}\right)_{\text {mean }}+\sum_{\text {Scatterers }}\left(\bar{H}_{\mathrm{d}}+\bar{H}_{\mathrm{r}}\right)
\end{aligned}
$$

where the subscripts $\mathrm{d}$ and $\mathrm{r}$ stand for the direct and reflected fields, respectively. $\bar{E}_{2}$ and $\bar{H}_{2}$, on the surface $\mathbf{S}$, are computed using the FDTD technique, described in the previous section. Since the fields computed by the forest model are in the frequency domain and the input to the FDTD code must be in time domain, a frequency-to-time-domain transformation is needed. Depending on the desired frequency range of simulation, the complex total field quantities from $f_{\text {start }}$ to $f_{\text {stop }}$ are computed over the FDTD box at $N$ discrete frequency points. The choice of $N$ (increments in frequency) depends on the size of the FDTD box or equivalently the alias-free time-domain period. Once the frequency-domain fields are computed, the timedomain representations are calculated by

$$
\begin{aligned}
& \bar{\varepsilon}_{1}(t)=2 \text { Real }\left\{\int_{f_{\text {start }}}^{f_{\text {stop }}} \bar{E}_{1 q} e^{-i 2 \pi f t} d f\right\} \\
& \bar{\zeta}_{1}(t)=2 \text { Real }\left\{\int_{f_{\text {start }}}^{f_{\text {stop }}} \bar{H}_{1 q} e^{-i 2 \pi f t} d f\right\} .
\end{aligned}
$$

To alleviate the effects of abrupt frequency truncations which results in high time-domain side lobes, a Gaussianweighting function can be introduced in (17). The output of FDTD simulation for tangential fields is in the time domain,

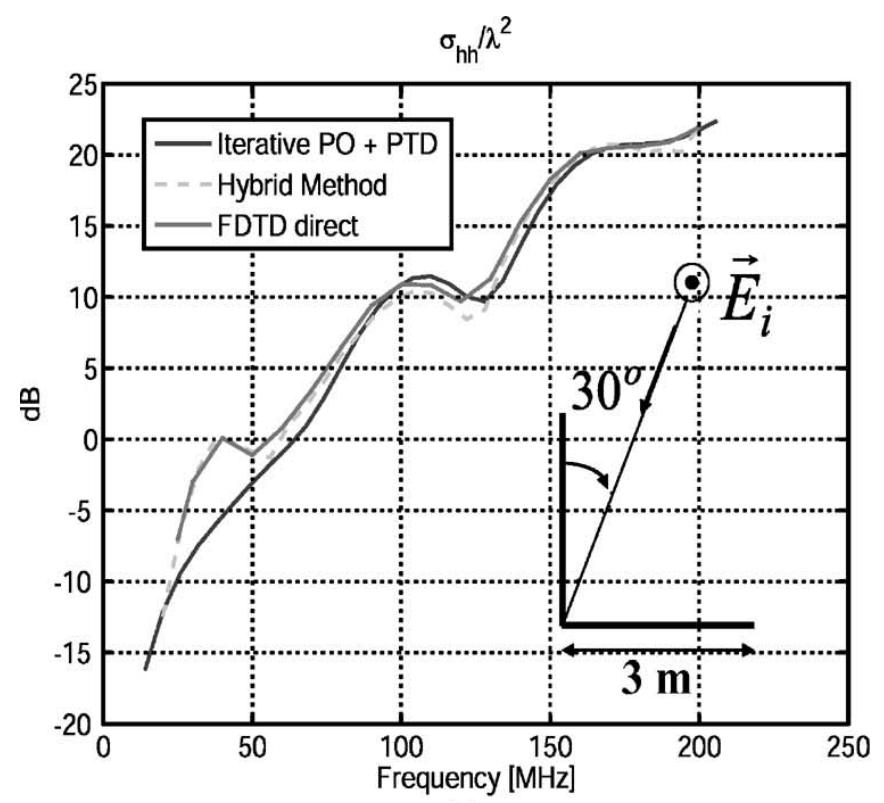

(a)

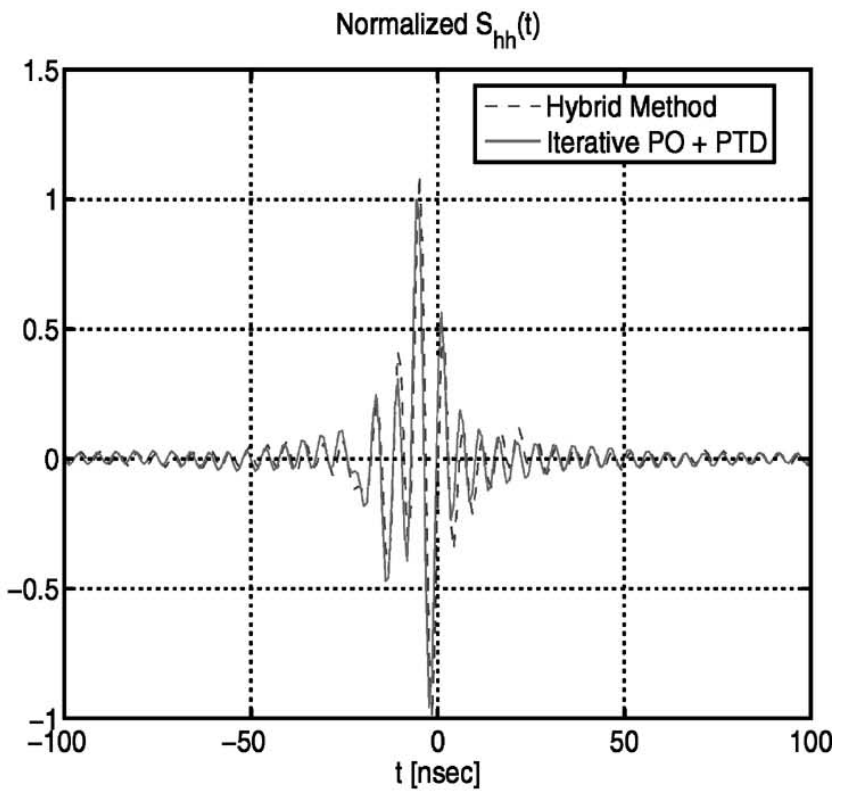

(b)

Fig. 3. Comparison of backscattering RCS from a dihedral corner reflector in free space, calculated by direct FDTD, hybrid method, and physical optics + PTD approximation for an $\mathrm{H}$-polarized incident plane wave propagating along $\theta_{i}=30^{\circ}$ and $\phi_{i}=180^{\circ}$. (a) Frequency domain. (b) Time domain.

and the fields quantities required in (15) are in the frequency domain. Denoting the time-domain response of the scattered fields computed by FDTD simulation by $\bar{\varepsilon}_{2}(t)$ and $\bar{\zeta}_{2}(t)$, the scattered fields in frequency domain are obtained from

$$
\begin{aligned}
\bar{E}_{2 q} & =\int_{-\infty}^{+\infty} \bar{\varepsilon}_{2}(t) e^{+i 2 \pi f t} d t \\
\bar{H}_{2 q} & =\int_{-\infty}^{+\infty} \bar{\zeta}_{2}(t) e^{+i 2 \pi f t} d t
\end{aligned}
$$




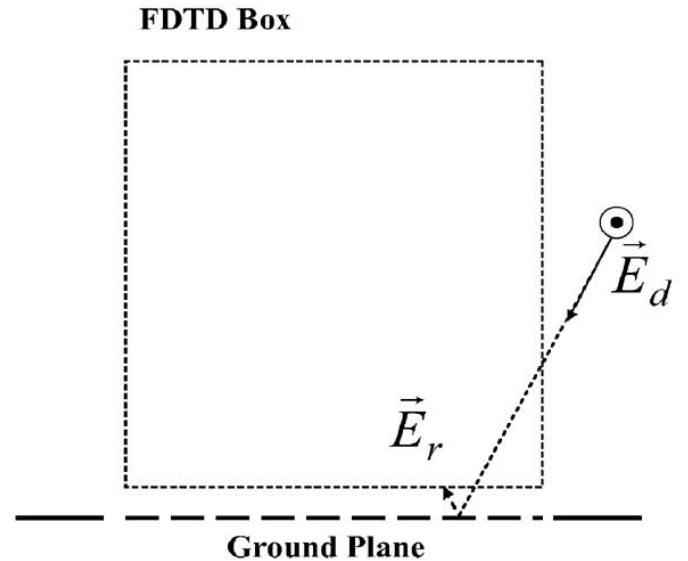

(a)

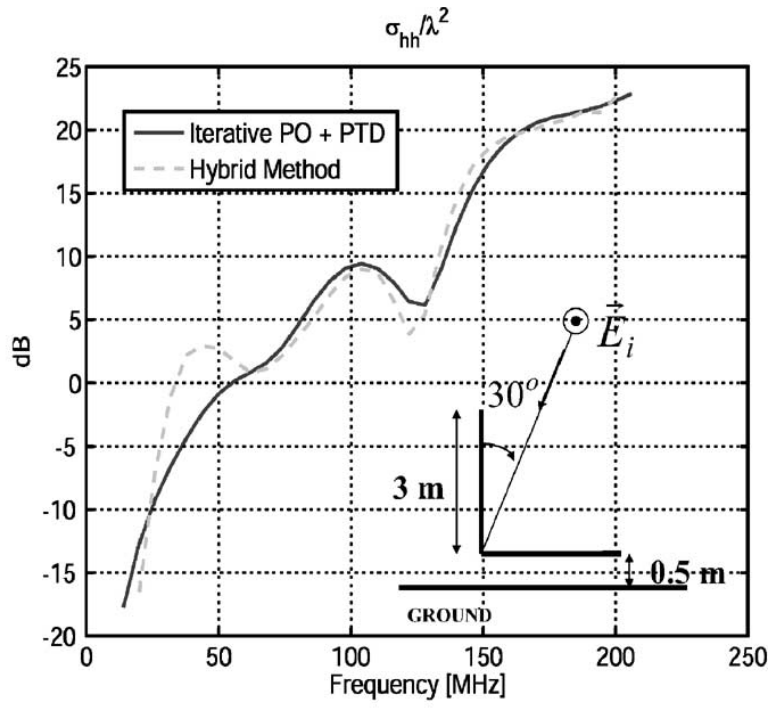

(b)

Fig. 4. (a) Geometry of the scattering problem when the effect of ground plane exists. (b) Comparison of backscattering RCS from a dihedral corner reflector, above a dielectric ground plane with $\epsilon_{\mathrm{r}}=5.62+i 0.94$, calculated by the hybrid method, and physical optics + PTD approximation for an H-polarized incident plane wave propagating along $\theta_{i}=30^{\circ}$ and $\phi_{i}=180^{\circ}$.

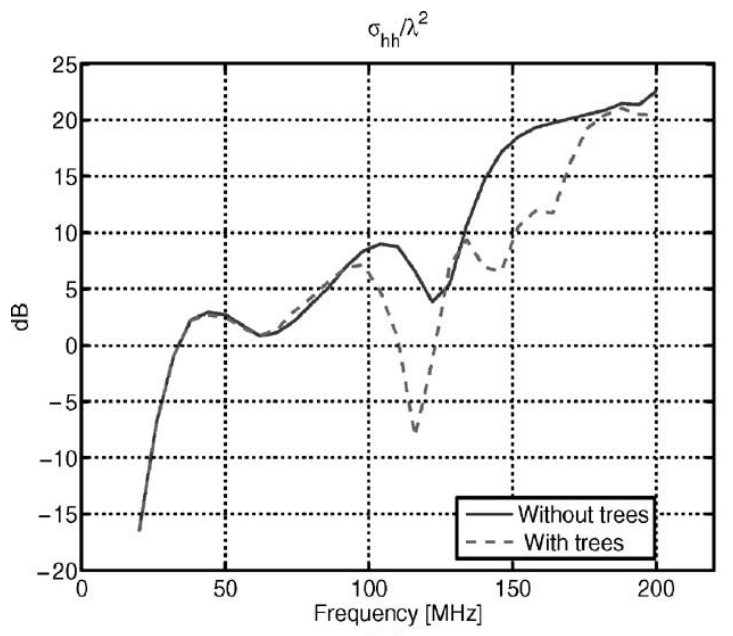

(a)

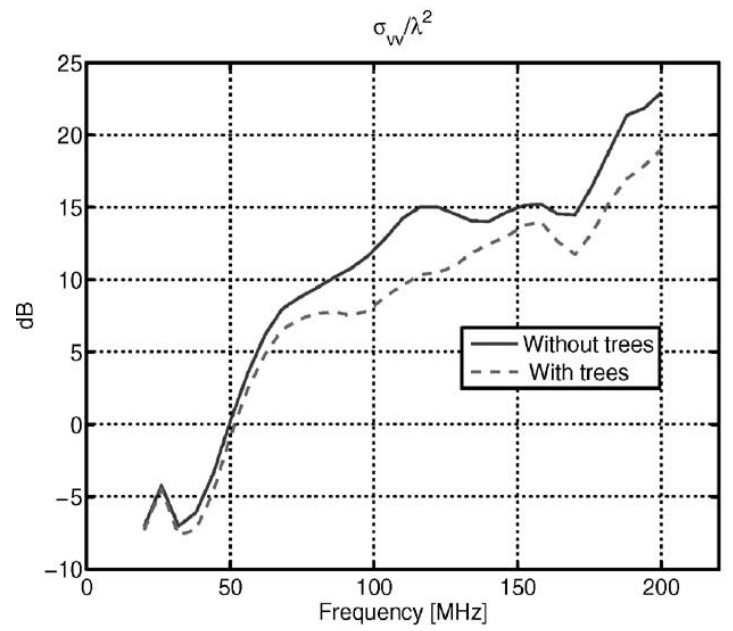

(c)

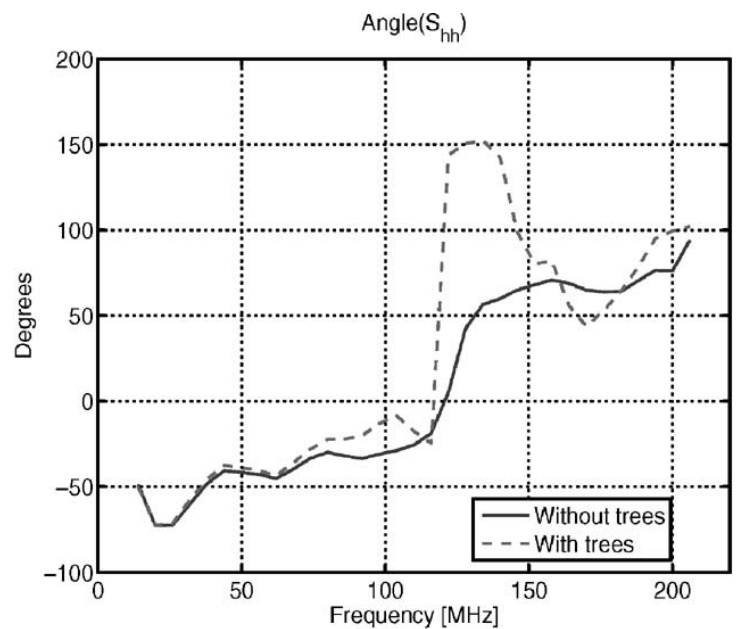

(b)

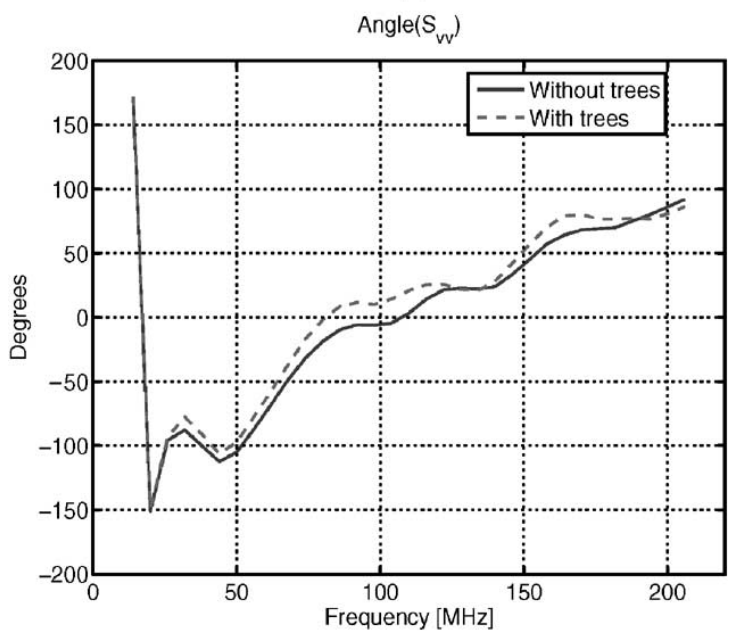

(d)

Fig. 5. Comparison of backscattering RCS from a dihedral corner reflector (each panel is $3 \mathrm{~m} \times 3 \mathrm{~m}$ ), placed $0.5 \mathrm{~m}$ above a dielectric ground plane with $\epsilon_{\mathrm{r}}=5.62+i 0.94$, calculated by the hybrid method, inside and outside of the forest for standard polarization configurations of incident plane wave propagating along $\theta_{i}=30^{\circ}$ and $\phi_{i}=180^{\circ}$. (a) $\sigma_{h h} / \lambda^{2}$. (b) $\operatorname{Angle}\left(S_{h h}\right)$. (c) $\sigma_{v v} / \lambda^{2}$. (d) $\operatorname{Angle}\left(S_{v v}\right)$. 
Expressions of (16) and (18) are used in (15) for the calculation of the backscattered field. It should be noted that, with this technique, only the first-order scattering interaction is taken into account. To demonstrate the validity of the proposed hybrid frequency-domain and time-domain method, a $3 \mathrm{~m} \times 3 \mathrm{~m}$ metallic dihedral corner reflector in free space is considered. A plane wave illumination is assumed and simulation is desired over a frequency range of 14-206 MHz. Since the target is inside an FDTD box with size of $3.96 \mathrm{~m} \times 3.96 \mathrm{~m} \times 3.96 \mathrm{~m}$, maximum frequency increment $\Delta f=43.74 \mathrm{MHz}$ is required to have alias-free time-domain response. For this simulation, we used $\Delta f=6 \mathrm{MHz}(N=33)$. The cell size in FDTD code is set to $12 \mathrm{~cm}$, which is smaller than $\lambda / 10$ at the highest simulation frequency. Due to the size of the FDTD box and cell size, a time span of about $212 \mathrm{~ns}$ with time step of $0.83 \mathrm{~ns}$ is considered in FDTD to provide an accurate solution. For the hybrid time- and frequency-domain approach first the incident field quantities are computed as a function of frequency over the FDTD box, and then the process described above was followed. Fig. 3 shows the backscatter RCS of the dihedral, using direct FDTD, the hybrid approach, and a totally independent approach using IPOs approximation and PTD [16]-[18]. Fig. 3(a) shows the RCS as a function of frequency for an H-polarized incident plane wave, propagating along $\theta_{i}=30^{\circ}$ and $\phi_{i}=180^{\circ}$. Fig. 3(b) shows the same result in time domain. Since the FDTD box is centered above the origin, a nonzero backscattered signal is also observed for negative times. As shown, an excellent agreement exists between direct FDTD and the hybrid approach. The small discrepancies are due to the effect of the discretizations in time, space, and frequency domains. Also, there is a very good agreement between the iterative $\mathrm{PO}+\mathrm{PTD}$ and the numerical results at high frequencies $(f>50 \mathrm{MHz})$ where the high-frequency approximation method is expected to work. A difficulty may arise due to the presence of the ground plane. The total incident field on the target is the superposition of the incident field and its reflection on the ground plane. In situations where the target is sufficiently above the ground plane, the superposition of the incident field and reflected field can be used as the total incident field on the target. However, in cases where the target is sitting on the ground, the target shadows the ground and no reflected fields from the shadowed region are generated. In this model, the effect of shadowing is accounted for, in an approximate manner by placing a perfectly absorbing layer on the ground over the shadowed area, as shown in Fig. 4(a). Fig. 4(b) shows a comparison of backscattering RCS from a dihedral corner reflector, above a dielectric ground plane with $\epsilon_{\mathrm{r}}=5.62+i 0.94$, calculated once using the proposed hybrid method then using PO + PTD approximation for an $\mathrm{H}$-polarized incident plane wave propagating along $\theta_{i}=30^{\circ}$ and $\phi_{i}=180^{\circ}$. A very good agreement is shown at high frequencies $(f>$ $60 \mathrm{MHz}$ ) where the iterative PO + PTD is expected to work properly.

\section{Numerical Simulations OF FOLIAGE-CAMOUFLAGED TARGETS}

To study the importance of foliage in modifying the RCS values of foliage-camouflaged targets, simulation of backscatter

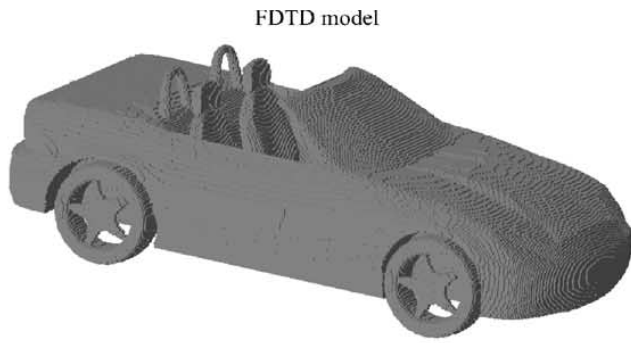

(a)

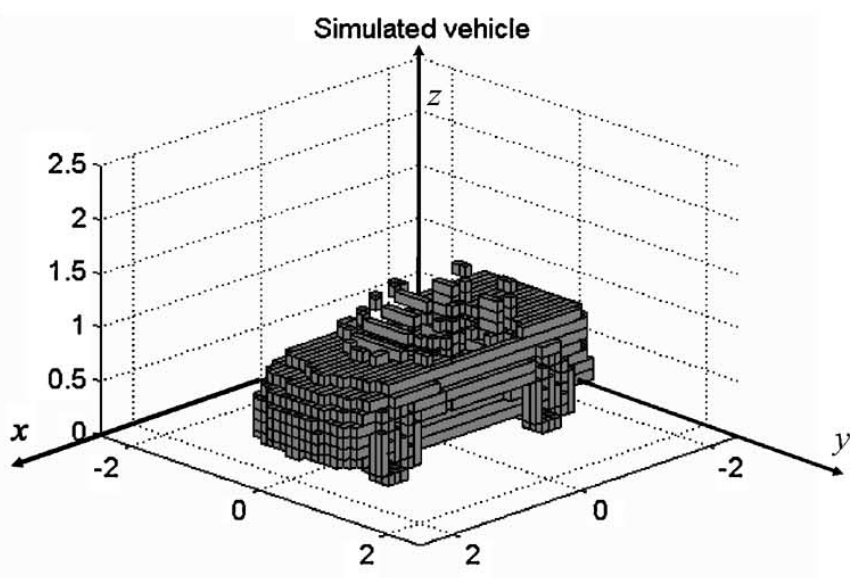

(b)

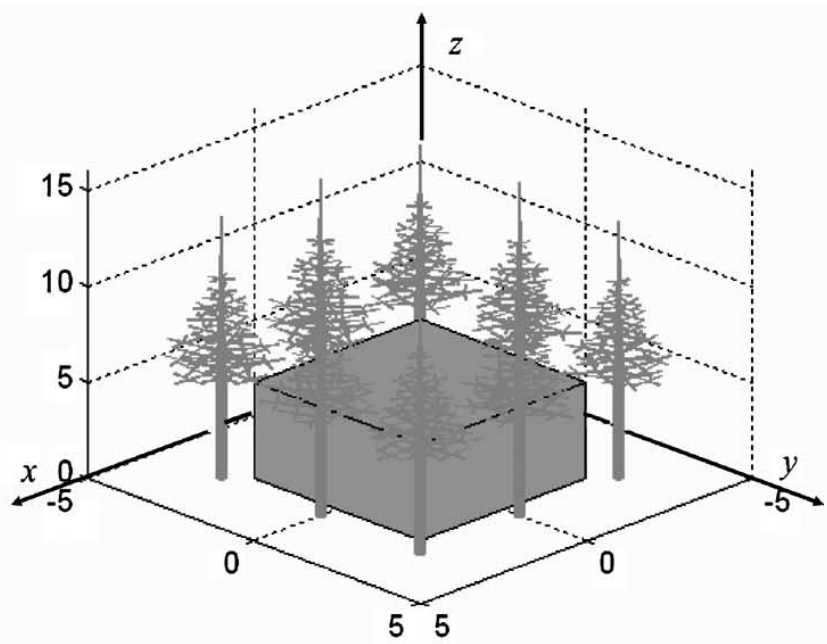

(c)

Fig. 6. (a) FDTD model of the hard target with a fine-resolution mesh (resolution $\approx 1 \mathrm{~cm}$ ), (b) course FDTD mesh (resolution $=10 \mathrm{~cm}$ ), used for VHF simulation, and (c) the FDTD box located inside a forest, including eight pine trees.

responses of metallic targets in a pine forest stand is examined. First, we consider the metallic dihedral corner reflector, used in the previous simulations (each panel is $3 \mathrm{~m} \times 3 \mathrm{~m}$ ). For this simulation the dihedral is placed $0.5 \mathrm{~m}$ above a lossy ground plane, having a relative permittivity of $\epsilon_{\mathrm{r}}=5.62+i 0.94$. It is assumed that the target is surrounded by eight nearby trees [see Fig. 6(c)]. These trees are generated by the statistical L-system having maximum height of $15.3 \mathrm{~m}$, crown radius of $3 \mathrm{~m}$, crown height of $9.6 \mathrm{~m}$, trunk radius of $10.9 \mathrm{~cm}$, and more than 5000 branches per tree. Since the target in the forest can 


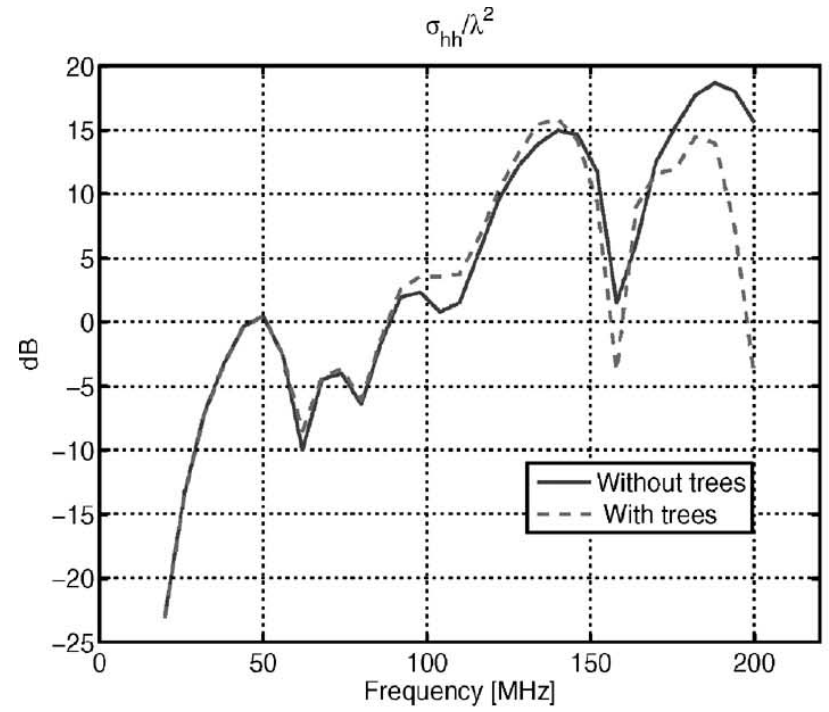

(a)

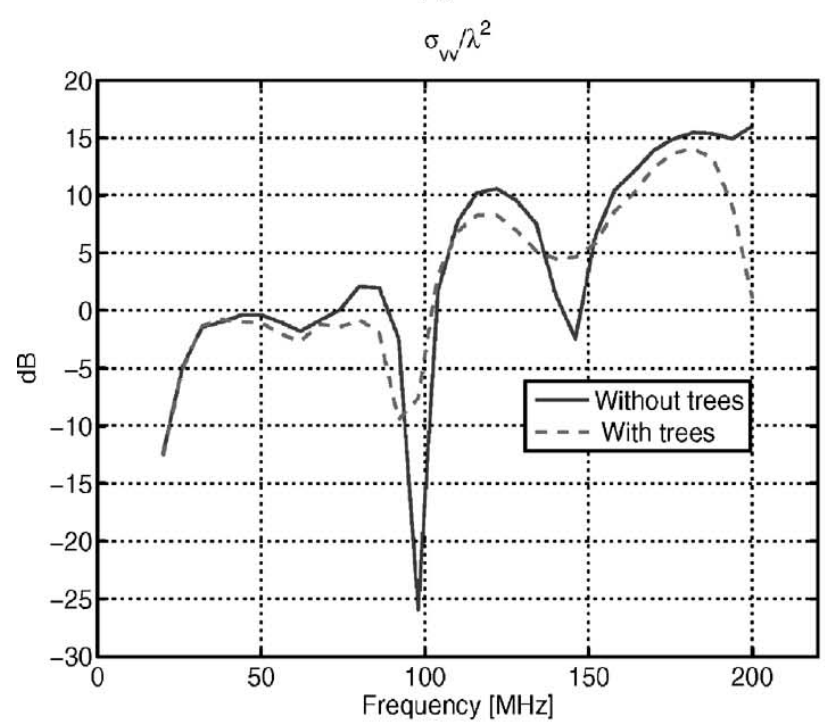

(c)

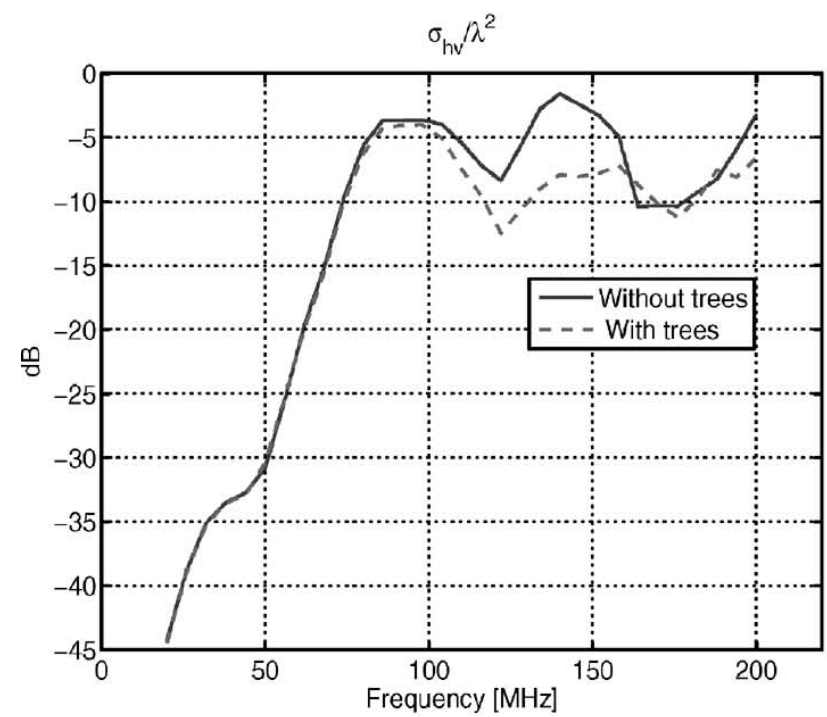

(e)

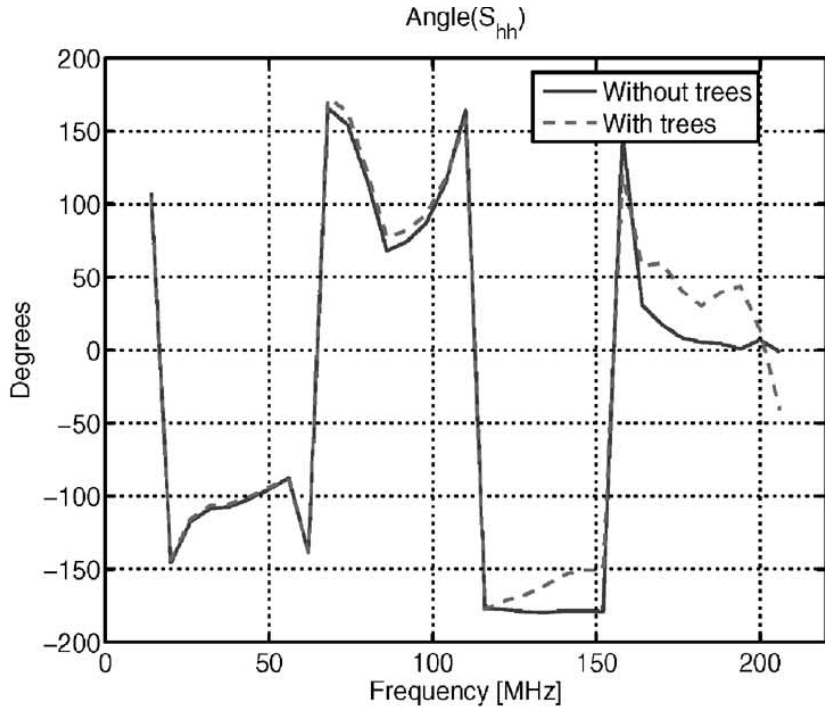

(b)

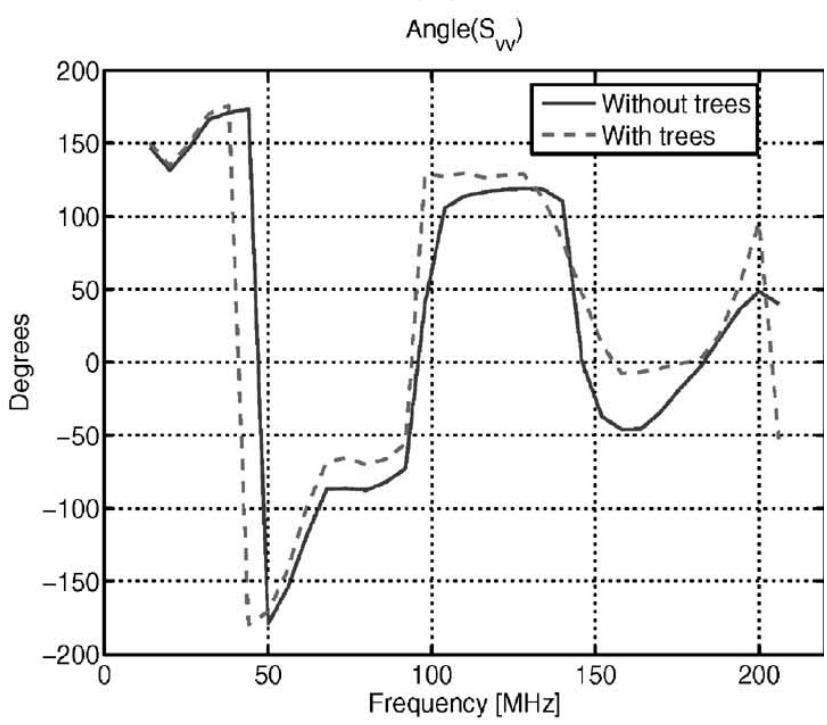

(d)

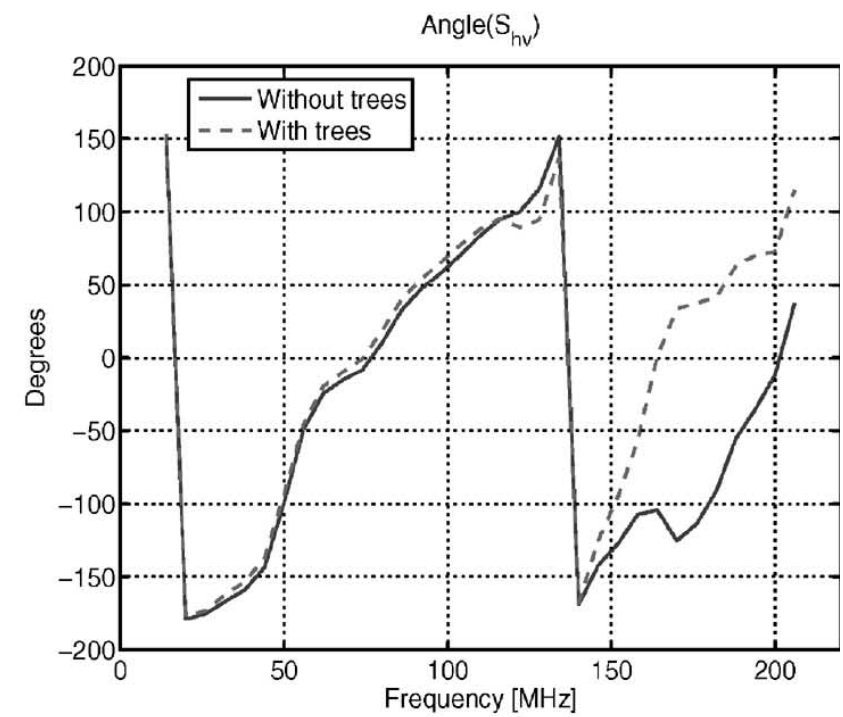

(f)

Fig. 7. Comparison of backscattering RCS from the metallic vehicle, above a dielectric ground plane with $\epsilon_{\mathrm{r}}=5.62+i 0.94$, calculated by the hybrid method, inside and outside of the forest for standard polarization configurations of incident plane wave propagating along $\theta_{i}=30^{\circ}$ and $\phi_{i}=180^{\circ}$. (a) $\sigma_{h h} / \lambda^{2}$. (b) Angle $\left(S_{h h}\right)$. (c) $\sigma_{v v} / \lambda^{2}$. (d) $\operatorname{Angle}\left(S_{v v}\right)$. (e) $\sigma_{h v} / \lambda^{2}$. (f) $\operatorname{Angle}\left(S_{h v}\right)$. 


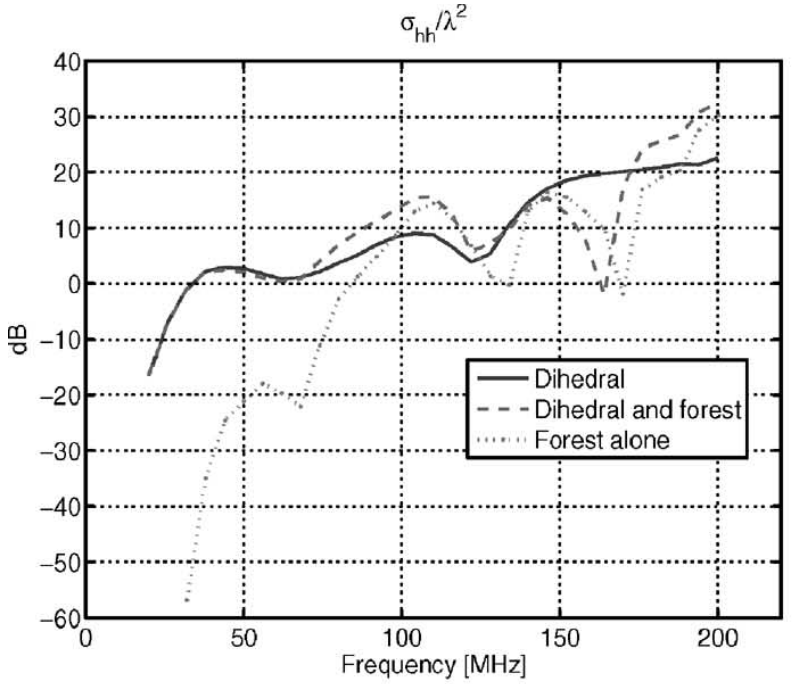

(a)

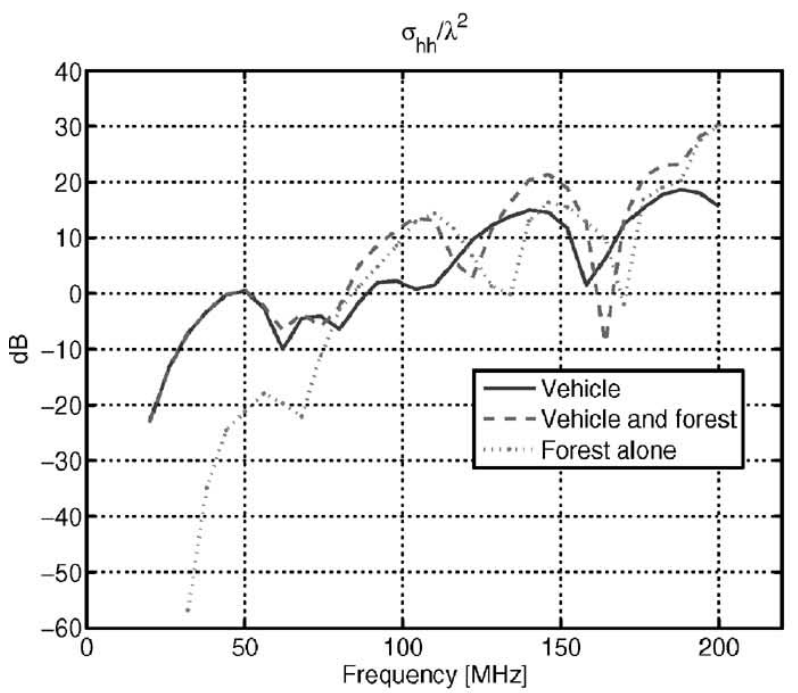

(c)

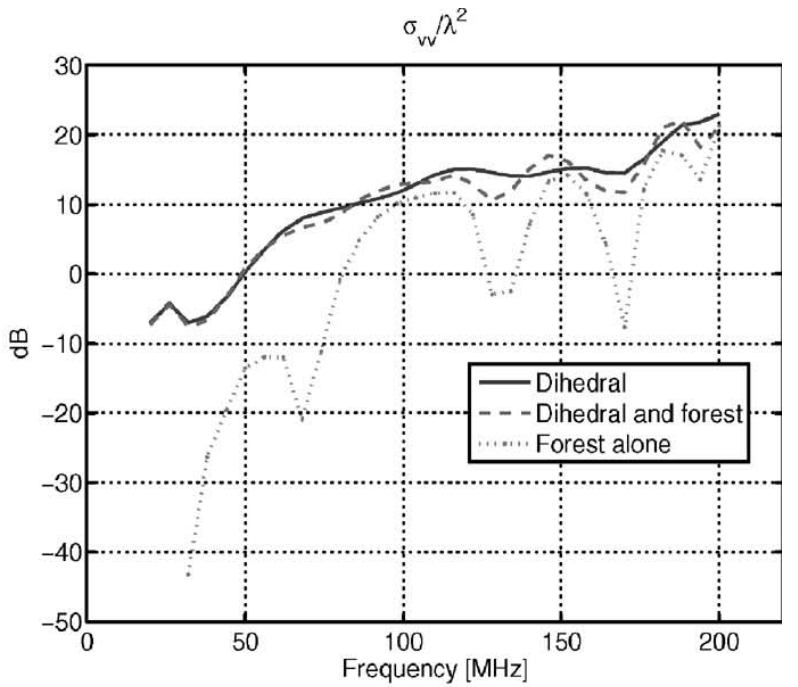

(b)

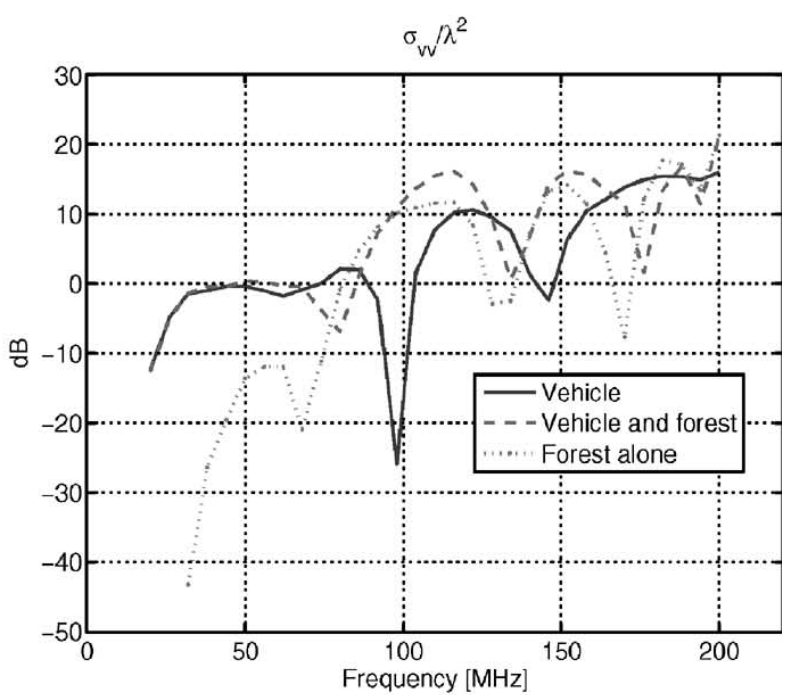

(d)

Fig. 8. Comparison of backscattering RCS from a target, target and forest, and forest alone, for horizontal and vertical polarizations of incident waves propagating along $\theta_{i}=30^{\circ}$ and $\phi_{i}=180^{\circ}$. (a) Dihedral $\sigma_{h h} / \lambda^{2}$. (b) Dihedral $\sigma_{v v} / \lambda^{2}$. (c) Vehicle $\sigma_{h h} / \lambda^{2}$. (d) Vehicle $\sigma_{v v} / \lambda^{2}$.

be fit within a volume with a maximum linear dimension of about $22.65 \mathrm{~m}$, the maximum frequency increment $\Delta f$ must be less than $13.25 \mathrm{MHz}$ to have alias-free time-domain response. For this example, the following parameters were chosen, $\Delta f=$ $6 \mathrm{MHz}$, cell size $=12 \mathrm{~cm}$, time span $=212 \mathrm{~ns}$, and time step $=$ $0.83 \mathrm{~ns}$. To make the code faster and at the same time observe accurate results, only scattering effects of branches that have radii more than $0.01 \lambda$ have been calculated. Other scatterers contribute only to the signal attenuation.

Fig. 5(a)-(d) shows the backscattered RCS response of the dihedral as a function of frequency once with and once without foliage, for basic radar polarization configurations. The backscatter responses of the target embedded in the foliage shown in these figures do not include the forest backscatter itself. As shown, at lower frequencies, the effect of forest on modifying the backscattered response of the target is negligible. However, for higher frequencies the effect of forest is observed in reducing and distorting the RCS pattern. At frequencies below $70 \mathrm{MHz}$, the foliage-scattering interaction contribution is much smaller than the dihedral scattering by itself and thus, in this case, the polarimetric-target signature does not change. For the same arrangement of the trees, a simulation is carried out for a complex metallic vehicle [see Fig. 6(a)] inside the FDTD box with dimensions of $5 \mathrm{~m} \times 5 \mathrm{~m} \times 5 \mathrm{~m}$ [see Fig. 6(c)], placed inside the forest. For this simulation, we used $\Delta f=6 \mathrm{MHz}$ $(N=33)$ and the cell size of $10 \mathrm{~cm}$. This surface of the FDTD box is composed of 62424 pixels for which the forest fields must be calculated. Due to the size of the FDTD box and cell size, a time span of about $176 \mathrm{~ns}$ with a time step of $0.69 \mathrm{~ns}$ is required for the FDTD technique to provide accurate results. Fig. 7(a)-(f) compares the frequency response of the backscatter from the target for standard polarization configurations, once with and once without foliage. As before the response of the target includes the foliage interaction (foliage-target and target foliage). The computation time for this simulation is estimated about three days, using two AMD Athlon processors, with 2-GHz CPU and 1-GB RAM. The scattering computation results provided here are run by personal computer for one 
realization of pine stand forest, including eight pine trees. In fact, using parallel computers, more number of realizations of forest can be simulated.

Practically, the radar received signal is the coherent summation of the target and the forest backscatter responses. The dihedral and the vehicle in previous simulations are used as targets inside the forest. Fig. 8 compares total backscattering from the target and the forest, the forest alone and the target, for horizontal and vertical polarizations of incident fields. Scattering from all eight pine trees are included in the forest response. As shown, the backscatter response of the camouflaged target is significantly distorted by the forest. It is observed that from frequencies above $60 \mathrm{MHz}$, the signature of the target response is changing which makes target detection very difficult. Target discrimination approaches, such as polarization signature and polarization optimization study are needed for identifying the target in a strong clutter environment.

\section{CONCLUSION}

At very low frequencies, scattering from the forest itself may be rather small, and the effect of forest on scattering behavior from the hard target can be carried out by applying a simple attenuation on RCS, and increase on propagation phase constant. However, at high frequencies the forest distorts the incident wave phase-front, as well as its polarization significantly. To show this, a hybrid foliage-target model is developed. The hybrid model is composed of two scattering models, the foliage model and the full-wave hard target scattering model. The foliage-scattering model uses the single-scattering theory applied to realistic looking tree structures generated by a fractal model known as L-systems. The target scattering is computed by FDTD. The connection between these two models, which accounts for the interaction between the foliage scatterers and the target, is carried out through the application of the reciprocity theorem. This model has the ability to characterize the scattering from camouflaged complex targets inside the forest canopy accurately. Simulations of scattering from hard targets embedded inside a forest canopy, in VHF band (20-200 MHz), was carried out using proposed hybrid model. Based on simulation results, it is observed that the signature response of a camouflaged vehicle with typical dimensions of $4 \mathrm{~m} \times 2 \mathrm{~m} \times$ $1.5 \mathrm{~m}$ inside a pine forest with density of about $0.05 \mathrm{trees} / \mathrm{m}^{2}$ is significantly changed for frequencies above $60 \mathrm{MHz}$. Clearly, the electric size of the target as well as forest parameters, such as density of the trees, determine this frequency.

\section{REFERENCES}

[1] L. M. Ulander, P.-O. Froelind, A. Gustavsson, H. Hellsten, and B. Larsson, "Detection of concealed ground targets in CARABAS SAR images using change detection," in Proc. Algorithms Synthetic Aperture Radar Imagery VI, Orlando, FL, Apr. 5-9, 1999, vol. 3721, pp. 243-252.

[2] L. M. H. Ulander, M. Blom, B. Flood, P. Follo, P.-O. Frolind, A. Gustavsson, T. Jonsson, B. Larsson, D. Murdin, M. Pettersson, U. Raaf, and G. Stenstrom, "Development of the ultra-wideband LORA SAR operating in the VHF/UHF-band," in Proc. IEEE Int. Geosci. Remote Sens., Jul. 21-25, 2003, vol. 7, pp. 4268-4270.

[3] K. Sarabandi, "Electromagnetic scattering from vegetation canopies," $\mathrm{Ph} . D$. dissertation, Dept. Elect. Eng. Comput. Sci., Univ. Michigan, Ann Arbor, 1989
[4] L. Tsang, J. A. Kong, and K.-H. Ding, Scattering of Electromagnetic Waves: Theories and Applications. Wiley Series in Remote Sensing. New York: Wiley, 2000.

[5] F. T. Ulaby, K. Sarabandi, K. McDonald, M. Whitt, and M. C. Dobson, "Michigan microwave canopy scattering model (MIMICS)," Univ. Michigan, Ann Arbor, Tech. Rep. 022486-T-1, Jul. 1988.

[6] M. A. Karam, A. K. Fung, R. H. Lang, and N. S. Chauhan, "A microwave scattering model for layered vegetation," IEEE Trans. Geosci. Remote Sens., vol. 30, no. 4, pp. 767-784, Jul. 1992.

[7] L. Tsang, J. A. Kong, and R. T. Shin, Theory of Microwave Remote Sensing. Wiley Series in Remote Sensing. New York: Wiley, 1985.

[8] L. Li, J. He, Z. Liu, X. Dong, and L. Carin, "MLFMA analysis of scattering from multiple targets in the presence of a half-space," IEEE Trans. Antennas Propagat., vol. 51, no. 4, pp. 810-819, Apr. 2003.

[9] S. R. Cloude, D. G. Corr, and M. L. Williams, "Target detection beneath foliage using polarimetric synthetic aperture radar interferometry," Waves Random Media, vol. 14, no. 2, pp. S393-S414, Apr. 2004.

[10] Y.-C. Lin and K. Sarabandi, "A Monte Carlo coherent scattering model for forest canopies using fractal-generated trees," IEEE Trans. Geosci. Remote Sens., vol. 37, no. 1, pp. 440-451, Jan. 1999.

[11] I.-S. Koh and K. Sarabandi, "Polarimetric channel characterization of foliage for performance assessment of GPS receivers under tree canopies," IEEE Trans. Antennas Propagat., vol. 50, no. 5, pp. 713-726, May 2002.

[12] K. Demarest, Z. Huang, and R. Plumb, "An FDTD near- to far-zone transformation for scatterers buried in startified ground," IEEE Trans. Antennas Propagat., vol. 44, no. 8, pp. 1150-1157, Aug. 1996.

[13] T. Dogaru and L. Carin, "Time-domain sensing of targets buried under a rough air-ground interface," IEEE Trans. Antennas Propagat., vol. 46, no. 3, pp. 360-372, Mar. 1998.

[14] H. Israelsson, L. M. H. Ulander, T. Martin, and J. I. H. Askne, "A coherent scattering model to determine forest backscattering in VHFband," IEEE Trans. Geosci. Remote Sens., vol. 38, no. 1, pp. 238-248, Jan. 2000.

[15] C. D. Moss, F. L. Teixeira, Y. E. Yang, and J. A. Kong, "Finite-difference time-domain simulation of scattering from objects in continuous random media," IEEE Trans. Geosci. Remote Sens., vol. 40, no. 1, pp. 178-186, Jan. 2002.

[16] M. Kaye, P. K. Murthy, and G. A. Thiele, "An iterative method for solving scattering problems," IEEE Trans. Antennas Propagat., vol. AP33, no. 11, pp. 1272-1279, Nov. 1985.

[17] T. Griesser and C. A. Balanis, "Backscatter analysis of dihedral corner reflectors using physical optics and physical theory of diffraction," IEEE Trans. Antennas Propagat., vol. AP-35, no. 10, pp. 1137-1147, Oct. 1987.

[18] F. Obelleiro, J. L. Rodriguez, and R. J. Burkholder, "An iterative physical optics approach for analyzing the electromagnetic scattering by large open-ended cavities," IEEE Trans. Antennas Propagat., vol. 43, no. 4, pp. 356-361, Apr. 1995.

[19] Y.-C. Lin, "A fractal-based coherent scattering and propagation model for forest canopies," Ph.D. dissertation, Dept. Elect. Eng. Comput. Sci., Univ. Michigan, Ann Arbor, 1997.

[20] M. L. Imhoff, "A theoretical analysis of the effect of forest structure on SAR backscatter and the remote sensing of biomass," IEEE Trans. Geosci. Remote Sens., vol. 33, no. 2, pp. 341-352, Mar. 1995.

[21] M. H. Zimmermann and C. L. Brown, Tree Structures and Function. New York: Springer-Verlag, 1971.

[22] A. Lindenmayer, "Developmental algorithms for multicellular organisms: A survey of L-systems," J. Theor. Biol., vol. 54, no. 1, pp. 3-22, Oct. 1975.

[23] B. Mandelbrot, Fractal Geometry of Nature. San Francisco, CA: Freeman, 1983.

[24] P. Prusinkiewicz and A. Lindenmayer, The Algorithmic Beauty of Plants. New York: Springer-Verlag, 1990.

[25] K. Sarabandi and T. B. A. Senior, "Low-frequency scattering from cylindrical structures at oblique incidence," IEEE Trans. Geosci. Remote Sens., vol. 28, no. 5, pp. 879-885, Sep. 1990.

[26] A. Taflove, Computational Electrodynamics: The Finite-Difference TimeDomain Method. Norwood, MA: Artech House, 1995.

[27] —_, Advances in Computational Electrodynamics: The FiniteDifference Time-Domain Method. Norwood, MA: Artech House, 1998.

[28] H. Mosallaei and Y. Rahmat-Samii, "Broadband characterization of complex periodic EBG structures: An FDTD/Prony technique based on the split-field approach," Electromagn. J., vol. 23, no. 2, pp. 135-151, Feb./Mar. 2003

[29] K. Sarabandi and P. F. Polatin, "Electromagnetic scattering from two adjacent objects," IEEE Trans. Antennas Propagat., vol. 42, no. 4, pp. 510-517, Apr. 1994.

[30] R. F. Harington, Time-Harmonic Electromagnetic Fields. New York: Wiley, 2001. 


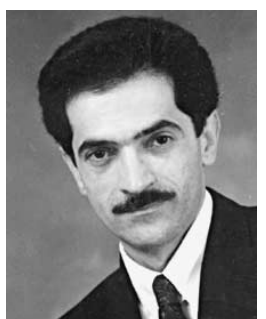

Kamal Sarabandi (S'87-M'90-SM'92-F'00) received the B.S. degree in electrical engineering from Sharif University of Technology, Tehran, Iran, in 1980, the M.S. degree in electrical engineering, in 1986, the M.S. degree in mathematics, and the Ph.D. degree in electrical engineering from The University of Michigan, Ann Arbor, in 1989.

He is currently the Director of the Radiation Laboratory and a Professor at the Department of Electrical Engineering and Computer Science, University of Michigan. His research areas of interest include microwave and millimeter-wave radar remote sensing, metamaterials, electromagnetic wave propagation, and antenna miniaturization. He has 20 years of experience with wave propagation in random media, communication channel modeling, microwave sensors, and radar systems and is leading a large research group including two research scientists, $12 \mathrm{Ph} . \mathrm{D}$., and two M.S. students. He has graduated $23 \mathrm{Ph} . \mathrm{D}$. and supervised numerous postdoctoral students. He has served as the Principal Investigator on many projects sponsored by the National Aeronautics and Space Administration, Jet Propulsion Laboratories, Army Research Office, Office of Naval Research, Applied Research Laboratory, National Science Foundation, Defense Advanced Research Projects Agency, and a larger number of industries. He has published many book chapters and more than 135 papers in refereed journals on EM-scattering, random media modeling, wave propagation, antennas, metamaterials, microwave measurement techniques, radar calibration, inverse scattering problems, and microwave sensors. He has also had more than 330 papers and invited presentations in many national and international conferences and symposia on similar subjects.

Dr. Sarabandi is a Vice President of the IEEE Geoscience and Remote Sensing Society (GRSS), and a member of IEEE Technical Activities Board Awards Committee. He served as the Associate Editor of the IEEE TRANSACTIONS ON ANTENNAS AND Propagation (AP) and the IEEE SENSORS Journal. He is also a member of Commissions $\mathrm{F}$ and D of the Union Radio Scientific International (URSI) and of The Electromagnetic Academy. He is listed in American Men and Women of Science Who's Who in America and Who's Who in Science and Engineering and was the recipient of the Henry Russel Award from the Regent of The University of Michigan (the highest honor The University of Michigan bestows on a faculty member at the assistant or associate level). In 1999, he received a German American Academic Council Distinguished Lecturer Award from the German Federal Ministry for Education, Science, and Technology given to about ten individuals worldwide in all areas of engineering, science, medicine, and law. He was also a recipient of a 1996 Electrical Engineering and Computer Science Department Teaching Excellence Award and a 2004 College of Engineering Research Excellence Award. In 2005, he received two prestigious awards, namely, the IEEE Geoscience and Remote Sensing Distinguished Achievement Award and The University of Michigan Faculty Recognition Award. In the past several years, joint papers presented by his students at a number of international symposia (IEEE Antennas and Propagation Society (APS)'95, '97, '00, '01, '03, '05; IEEE International Geoscience and Remote Sensing Symposium (IGARSS)' 99, '02, IEEE International Microwave Symposium (IMS)'01, United States National Committee (USNC) International Union for Radio Science (URSI)'04, '05, '06) have received Best Student Prize Paper Awards.

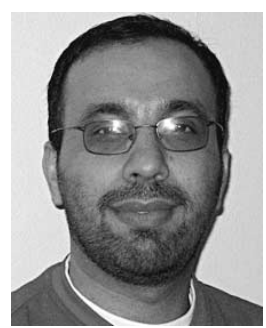

Mojtaba Dehmollaian (S'04) was born in Iran in 1978. He received the B.S. and M.S. degrees in electrical engineering from the University of Tehran, Tehran, Iran, in 2000 and 2002, respectively. He is currently working toward the Ph.D. degree at The University of Michigan, Ann Arbor.

His research interests are in the area of applied computational EMs with particular interest in remote sensing.

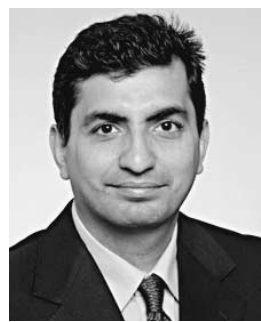

Hossein Mosallaei (S'98-M'02-SM'02) received the B.Sc. and M.Sc. degrees in electrical engineering from Shiraz University, Shiraz, Iran, in 1991 and 1994, respectively, and the Ph.D. degree in electrical engineering from the University of California, Los Angeles, in 2001.

$\mathrm{He}$ is currently an Assistant Professor of electrical and computer engineering with the College of Engineering, Northeastern University, Boston, MA. From 2002 to 2005 , he was on the faculty of the Department of Electrical Engineering and Computer Science, University of Michigan, Ann Arbor, as an Assistant Research Scientist. $\mathrm{He}$ served as the Vice-Chair of the IEEE Michigan Trident Chapter (AP, Microwave Theory and Techniques, Electron Devices) in 2005. He is the author or coauthor of more than 70 technical journal articles and conference papers. $\mathrm{He}$ is the holder of one U.S. patent and has one pending patent. His research interests include applied electromagnetics and radio-frequency circuits with focus on metamaterials and microscale/nanoscale devices and systems.

Dr. Mosallaei is a Full Member of the International Scientific Radio Union (URSI) and a member of the American Association for the Advancement of Science. He has organized several special sessions in various IEEE conferences and has been a plenary session speaker in many national and international symposia. He was the recipient of the student prize paper awards in APS 2000, 2001, 2003, and 2005 with his student; the URSI Young Scientist Award in 2001; and the Raj Mittra Travel Grant Award in 2002. He is listed in Who's Who in America. 